\title{
Reentrant Phase Transitions of the Blume-Emery-Griffiths Model for a Simple Cubic Lattice on the Cellular Automaton
}

\author{
N. Seferoğlu* and B. Kutlu** \\ * Gazi Üniversitesi, Fen Bilimleri Enstitüsü, Fizik Anabilim Dalı, \\ Ankara, Turkey \\ ** Gazi Üniversitesi, Fen -Edebiyat Fakültesi, Fizik Bölümü, \\ 06500 Teknikokullar, Ankara, Turkey \\ e-mail: nurguls@gazi.edu.tr \\ e-mail: bkutlu@gazi.edu.tr
}

\begin{abstract}
The spin-1 Ising (BEG) model with the nearest-neighbour bilinear and biquadratic interactions and single-ion anisotropy is simulated on a cellular automaton which improved from the Creutz cellular automaton(CCA) for a simple cubic lattice. The simulations have been made for several sets of parameters $K / J$ and $D / J$ in the $-3<D / J \leq 0$ and $-1 \leq K / J \leq 0$ parameter regions. The re-entrant and double re-entrant phase transitions of the BEG model are determined from the temperature variations of the thermodynamic quantities $(M, Q$ and $\chi)$. The phase diagrams characterizing phase transitions are compared with those obtained from other methods.

Keywords: spin-1 Ising model; Creutz cellular automaton; re-entrant phase transition; simple cubic lattice.

*Corresponding author
\end{abstract}




\section{Introduction}

The Blume-Emery-Griffiths (BEG) $\operatorname{model}^{1}$ was originally introduced in order to explain the phase separation and superfluidity in the $\mathrm{He}^{3}-\mathrm{He}^{4}$ mixtures. Subsequently, the model was used in the description of a variety of different physical phenomena such as multicomponent fluids ${ }^{2}$, microemulsions $^{3}$, and semiconductors alloys ${ }^{4}$, etc.

The Hamiltonian of the BEG model is given by,

$$
H_{I}=J \sum_{<i j>} S_{i} S_{j}+K \sum_{<i j>} S_{i}^{2} S_{j}^{2}+D \sum_{i} S_{i}^{2}
$$

where $s_{i}= \pm 1,0$ and $\langle i j>$ denotes summation over all nearest-neighboring (nn) spin pairs on a simple cubic lattice. The parameters of $J$ and $K$ are the bilinear and biquadratic interaction energies, respectively and $D$ is the single-ion anisotropy constant. The three-dimensional BEG model has been extensively studied by different techniques, using the mean-field approximation $(\mathrm{MFA})^{(1,5-7)}$, effective-field theory ${ }^{(8-11)}$, two-particle cluster approxima-

tion (TPCA $)^{12}$, Bethe approximation ${ }^{13}$, high-temperature series expansion ${ }^{14}$, renormalization group theory ${ }^{15}$, Monte Carlo simulations ${ }^{(13,16-17)}$, linear chain $\operatorname{approximation}^{(18,19)}$ and cellular automaton ${ }^{(20,21)}$

In this paper we studied the three-dimensional BEG model using an improved heating algorithm from the Creutz Cellular Automaton (CCA) for simple cubic lattice. The CCA algorithm is a microcanonical algorithm interpolating between the canonical Monte Carlo and molecular dynamics techniques on a cellular automaton, and it was first introduced by Creutz ${ }^{22}$. In the previous papers ${ }^{(20,21,23-26)}$, the CCA algorithm and improved algo- 
rithms from CCA were used to study the critical behavior of the different Ising model Hamiltonians on the two and three-dimensions. It was shown that they have successfully produced the critical behavior of the models.

The BEG model has a complicated phase diagrams and has several kinds of phase transitions, such as re-entrant and double re-entrant transitions for some values of the model parameters on the three-dimensional lattices. However, there exists the differences between the phase diagrams in the $\left(d=D / J, k=K / J, t=k_{B} T / z J\right)$ plane is obtained for certain parameter values ${ }^{(6,12,13,16)}$, where $z$ is the coordination number. Recently, TPCA $^{12}$ calculations of the three dimensional BEG model with the ferromagnetic bilinear interaction show that the phase diagrams are qualitatively different from the phase diagrams obtained with the $\mathrm{MFA}^{6}$ and Bethe approximation ${ }^{13}$ for the $k=-0.5$ parameter. In addition, the order parameters exhibit a different critical behavior for the double re-entrant phase transition. Thus, the alternative results are needed to check the differences in results. The aim of the present paper is to study re-entrant behaviors of the BEG model in the case of $J<0$ and to obtain the phase diagrams for several sets of parameters $k$ and $d$ in the $-3<d \leq 0$ and $-1 \leq k \leq 0$ parameter regions. For this purpose, the thermodynamic quantities are computed on a simple cubic lattice with linear dimension $L=18$ and the finite critical temperatures are estimated from the maxima of the susceptibility. The model is explained in Sec.2, the results are discussed in Sec.3 and a conclusion is given in Sec.4.

\section{Model}

Three variables are associated with each site of the lattice. The value of 
each sites are determined from its value and those of its nearest-neighbors at the previous time step. The updating rule, which defines a deterministic cellular automaton, is as follows: Of the three variables on each site, the first one is Ising spin $B_{i}$. Its value may be 0 or 1 or 2 . The Ising spin energy for the model is given by Eq.1. In Eq.1, Si=Bi-1. The second variable is for momentum variable conjugate to the spin (the demon). The kinetic energy associated with the demon, $H_{k}$, is an integer, which equal to the changing in the Ising spin energy for the any spin flip and its values lie in the interval $(0$, $\mathrm{m})$. The upper limit of the interval, $\mathrm{m}$, is equal to $24 \mathrm{~J}$. The total energy

$$
H=H_{I}+H_{K}
$$

is conserved.

The third variable provides a checkerboard style updating, and so it allows the simulation of the Ising model on a cellular automaton. The black sites of the checkerboard are updated and then their color is changed into white; white sites are changed into black without being updated. The updating rules for the spin and the momentum variables are as follows: For a site to be updated its spin is changed one of the other two states with $1 / 2$ probability and the change is transferable to or from the momentum variable associated with this site, such that the total energy $H$ is conserved, then this change is done and the momentum is appropriately changed. Otherwise the spin and the momentum are not changed.

For a given total energy the system temperature is obtained from the average value of kinetic energy, which is given by: 


$$
<E>=\frac{\sum_{n=0}^{m} n e^{-n J / k T}}{\sum_{n=0}^{m} e^{-n J / k T}}
$$

where $E=H_{K}$. The expectation value in $E q .3$ is a average over the lattice and the number of the time steps. Because of the third variable, the algorithm requires two time steps to give every spin of the lattice a change to change. Thus, in comparison to ordinary Monte Carlo simulations, two steps correspond to one full sweep over the system variables.

The heating algorithm is divided into two basic parts, initialization procedure and the taking of measurements. In the initialization procedure, firstly, all spins in the lattice sites take the ferromagnetic ordered structure $(\uparrow \uparrow)$ and the kinetic energy per site which is equal to the maximum change in the Ising spin energy for the any spin flip is given to the certain percent of the lattice via the second variables. This configuration is run during the 10.000 cellular automaton time steps. At the end of the this step, the configuration in the ordered structure at the low temperature is obtained. In the next steps last configuration in the ordered structure has been chosen as a starting configuration for the heating run. Rather than resetting the starting configuration at each energy, it is used the final configuration at a given energy as the starting point for the next. During the heating cycle, energy is added to the system through the second variables $\left(H_{k}\right)$ after 1.000 .000 cellular automaton steps.

\section{Results and discussion}

The three-dimensional BEG model is simulated with the heating algo- 
rithm which improved from the Creutz Cellular Automaton. The simulations were executed on simple cubic lattice $L \mathrm{x} L \mathrm{x} L$ of the linear dimensions $L=18$ with periodic boundary conditions. The computed values of the quantities are averages over the lattice and over the number of time steps (1.000.000) with discard of the first 100.000 time steps during which the cellular automaton develops. Hereafter we shall use the terminology of Ref. 13 for the definition of phases: $\mathrm{F}$, the ferromagnetic phase $(M \neq 0, Q \neq 2 / 3)$; $\mathrm{Q}$, the quadrupolar phase $(M=0, Q \neq 2 / 3)$.

In the ground state, the phase diagram of the BEG model on the $(d, k)$ plane for $-1 \leq k \leq 0$ parameter region is shown in Fig.1. The region of the perfect zero ordering and ferromagnetic ordering are separated by the line ${ }^{13}$ $k=-1-d / 3$ represented with the solid line. The types of phase transition (PT) obtained from the CCA calculations are also shown in Fig.1. In the ferromagnetic ordering region, the $Q \rightarrow F$ phase transitions occur according to the values of $k$ and $d$. However, the re-entrant $Q \rightarrow F \rightarrow Q$ and double re-entrant $Q \rightarrow F \rightarrow Q \rightarrow F$ phase transitions take place for some values of $k$ and $d$ near the phase boundary.

For the reproduce of the phase diagram using the CCA simulations, the temperature variation of the order parameters $(M$ and $Q)$ and the susceptibility $(\chi)$ are calculated at $d=0,-0.25,-1$ and -2.5 for several $k$ values on the finite lattice with $L=18$. For $d=0$, the order parameters and the susceptibility data are shown in Fig.2 (a) and (b). There is the second order phase transition from quadrupolar phase to ferromagnetic phase $(Q \rightarrow F)$ at $d=0$ for $k \geq-1$. In this region, the susceptibility data shows characteristic 
peaks at the critical temperature. However for $k=-1$ the value of the magnetization at the lowest temperature is much lower than the magnetization for the $k>-1$ values.

The order parameters $M$ and $Q$ obtained at $d=-0.25$ for different $k$ values are shown in Fig.3 (a) and (b). For $k \geq-0.90$, there is only one second order $Q \rightarrow F$ phase transition in the system. In the interval $-0.95 \leq k<-0.90$, the re-entrant $Q \rightarrow F \rightarrow Q$ phase transitions take place and both of them are second order. At the same time, the data of the susceptibility shows the two peaks which belong the $Q \rightarrow F$ and $F \rightarrow Q$ phase transitions in the $-0.95 \leq k<-0.90$ parameter region [Fig. 3(c)].

For $d=-1$, the temperature dependence of the order parameters and the susceptibility are given in Fig.4. As seen from Fig.4, while the secondorder $Q \rightarrow F$ phase transition occurs for $k>-0.66$, the double re-entrant $Q \rightarrow F \rightarrow Q \rightarrow F$ phase transitions occur for $k=-0.66,-0.67$ and -0.68 . For $k=-0.66$, only the higher transition $Q \rightarrow F$ is second order and the others are first order. At the same time, the susceptibility for the lower and middle transitions show a sharp peaks at two different transition temperature [Fig.4 (c)]. Nevertheless, for $k=-0.67$ and -0.68 only the lower phase transition is first order because the order parameters exhibit a discontinuous behavior and the susceptibility exhibit a jump. According to these results, the middle phase transition $F \rightarrow Q$ in the double re-entrant $Q \rightarrow F \rightarrow Q \rightarrow F$ phase transition can be the first order and second order. This is in agreement with the results obtained by the $\mathrm{MFA}^{6}$ and $\mathrm{TPCA}^{12}$ but disagreement with Bethe approximation(BA $)^{13}$ which gives only the lower 
phase transition can be first order.

In Fig.5, the order parameters and the susceptibility are shown for different values of the $k$ parameter at $d=-2.5$. The re-entrant or double re-entrant PT's are not seen for $d=-2.5$. While the $Q \rightarrow F$ phase transition is second order for $k \geq-0.12$, the $Q \rightarrow F$ phase transition is first order for $k<-0.12$. Nevertheless, the susceptibility shows a characteristic behavior for $k \geq-0.12$ while a jump in the susceptibility appears for $k<-0.12$.

Consequently, while the $Q \rightarrow F$ PT take place at $d=0$ for $k \geq-1$, the re-entrant $Q \rightarrow F \rightarrow Q$ and double re-entrant $Q \rightarrow F \rightarrow Q \rightarrow F$ PT's occur at $d=-0.25,-0.5,-1$ and -1.5 for certain values of $k$. The representative phase diagrams relevant to the these phenomena are shown in Fig.6. The phase diagrams obtained at $d=0$ and -1.5 represent similar behavior with the Bethe approximation results ${ }^{13}$ illustrated in Fig.6.

Finally, we obtain the $(d, t)$ phase diagram for $k=-0.5$ [Fig.7]. It is quite different from the phase diagram obtained with different methods ${ }^{(6,15)}$ at $k=-0.5$. In the phase diagram obtained with the $\mathrm{MFA}^{6}$ and renormalization group theory ${ }^{15}$ for $k=-0.5$, the second order PT line terminates at the critical end point E at the first order PT line and the first order PT line terminates at the critical point $\mathrm{C}$ inside the ferromagnetic phase. On the other hand, there is a tricritical point in the phase diagram obtained with TPCA $^{12}$ for $k=-0.5$. As seen Fig.7, there is no critical point $\mathrm{C}$ in the ferromagnetic phase and only a tricritical point exists in the phase diagram with CCA calculations as in TPCA. 


\section{Conclusion}

The BEG model is simulated using the heating algorithm of the cellular automaton for simple cubic lattice. The CCA calculations show that the BEG model exhibits the re-entrant and double re-entrant phase transitions for some values of the $k$ and $d$ parameters near the phase boundary as expected. According to the Bethe approximation ${ }^{13}$, the lower PT in the double reentrant $Q \rightarrow F \rightarrow Q \rightarrow F$ PT is of the first order and the other PT's are of the second order. Contrast to this, the $\mathrm{MFA}^{6}$ and $\mathrm{TPCA}^{12}$ calculations suggest that the middle PT can be also the first order. The CCA results are compatible with the MFA and TPCA results at this point. We have also reconstructed the phase diagrams in the $(d, k, t)$ parameters space using the CCA simulations. The phase diagrams in the $(k, t)$ plane for $d=0$ and -1.5 are similar to the phase diagrams obtained by the $\mathrm{BA}^{13}$ and $\mathrm{TPCA}^{12}$ at those parameters. Although the $\mathrm{MFA}^{6}$ and renormalization group theory ${ }^{15}$ predict that the $(d, t)$ phase diagram for $k=-0.5$ has an end point at the first order phase transition line and a critical point in the ferromagnetic phase, the CCA calculations for $k=-0.5$ indicate that there is only a tricritical point as the results of $\mathrm{TPCA}^{12}$.

\section{Acknowledgements}

This work is supported by a grant from Gazi University(BAP:05/200307).

\section{References}

[1] M. Blume, V.J. Emery and R.B. Griffiths, Phys. Rev. A 4 (1971) 1071 . 
[2] J. Lajzerowicz, J. Sivardiere, Phys. Rev. A 11 (1975) 2079; J. Sivardiere, J. Lajzerowicz, Phys. Rev. A 11 (1975) 2090.

[3] M. Schick, W. H. Shih, Phys. Rev. B 34 (1986) 1797.

[4] K. E. Newman, J. D. Dow, Phys. Rev. B 27 (1983) 7495.

[5] H.H. Chen and P. M. Levy, Phys. Rev.B 7, (1973) 4267.

[6] W. Hoston and A. N. Berker, Phys. Rev. Lett. 67 (1991) 1027.

[7] J. A. Plascak, J. G. Moreira and F. C. Sa Barreto, Phys. Lett. A 173 (1993) 360 .

[8] T. Kaneyoski and E. F. Sarmento, Physica A 152, (1988) 343.

[9] J.W. Tucker, J. Magn. Magn. Mater 87 (1990) 16.

[10] K. G. Chakraborty, Phys. Rev. B 29 (1984) 1454.

[11] A. F. Siqueira and I. P. Fittipaldi, Phys. Rev. B 31 (1985) 6092.

[12] O. R. Baran and R. R. Levitski, Phys. Rev. B 65 (2002) 172407.

[13] K. Kasono and I. Ono, Z. Phys. B: Cond. Matter 88 (1992) 205.

[14] D.M. Saul, M. Wortis and D. Stauffer, Phys.Rev. B 9 (1974) 4964.

[15] R. R. Netz and A. N. Berker, Phys.Rev. B 47 (1993) 15019.

[16] O. F. Alcantara Bonfirm and C. H. Obcemea, Z. Phys. B: Cond. Matter 64 (1986) 469.

[17] D. Wimgert and D.Stauffer, Physica A 219 (1995) 135.

[18] J. A. Plascak, N. P. Silva, Phys. Stat. Sol. (b) 110 (1982) 669.

[19] E. Albayrak, M. Keskin, J. Magn. and Magn. Materials 203 (2000) 201.

[20] B. Kutlu, A. Özkan, N. Seferoğlu, A. Solak and B. Binal, Int. J. Mod. Phys. C 16 (2005) 1933. 
[21] A. Özkan, N. Seferoğlu and B. Kutlu, Physica A 362 (2006) 327.

[22] M. Creutz, Phys. Rev. Lett. 50 (1983) 1411.

[23] B. Kutlu, Physica A 234 (1997) 807.

[24] B. Kutlu, Int. J. Mod. Phys. C 12, (2001) 1401

[25] B. Kutlu, Int. J. Mod. Phys. C 14, (2003) 1305

[26] N. Aktekin, Annual Reviews of Computational Physics VII, ed. D. Stauffer (World Scientific, Singapore, 2000), pp. 1-23.

Figure Captions

Fig.1. The phase diagram ( $d, k$ plane) in the ground state and the types of phase transitions obtained on a simple cubic lattice.

Fig.2. The temperature dependence of (a) the order parameters $M$ and $Q$, (b) the susceptibility $\chi$ for $d=0$.

Fig.3. The temperature dependence of (a) the order parameter $M, \mathrm{~b}) Q$, (c) the susceptibility $\chi$ for $d=-0.25$.

Fig.4. The temperature dependence of (a) the order parameters $M, \mathrm{~b}$ ) $Q$, (c) the susceptibility $\chi$ for $d=-1$.

Fig.5. The temperature dependence of (a) the order parameters $M$ and $Q$, (b) the susceptibility $\chi$ for $d=-2.5$.

Fig.6. The phase diagrams in the $(k, t)$ plane for values of the $d=0$, $-0.25,-0.5,-1$ and -1.5 . The closed and open symbols represent the secondorder and first-order phase transitions for the CCA calculations, respectively. The dashed and thick dashed lines indicate the second-order and first-order phase transitions for the Bethe approximation $(\mathrm{BA})^{13}$ at the $d=0$ and -1.5 parameter values. 
Fig.7. The phase diagram in the $(d, t)$ plane for $k=-0.5$. The solid line and dotted line represent the second-order and first-order phase transitions for TPCA ${ }^{12}$ calculations. The closed and open squares represent the secondorder and first-order phase transitions for CCA calculations, respectively. 


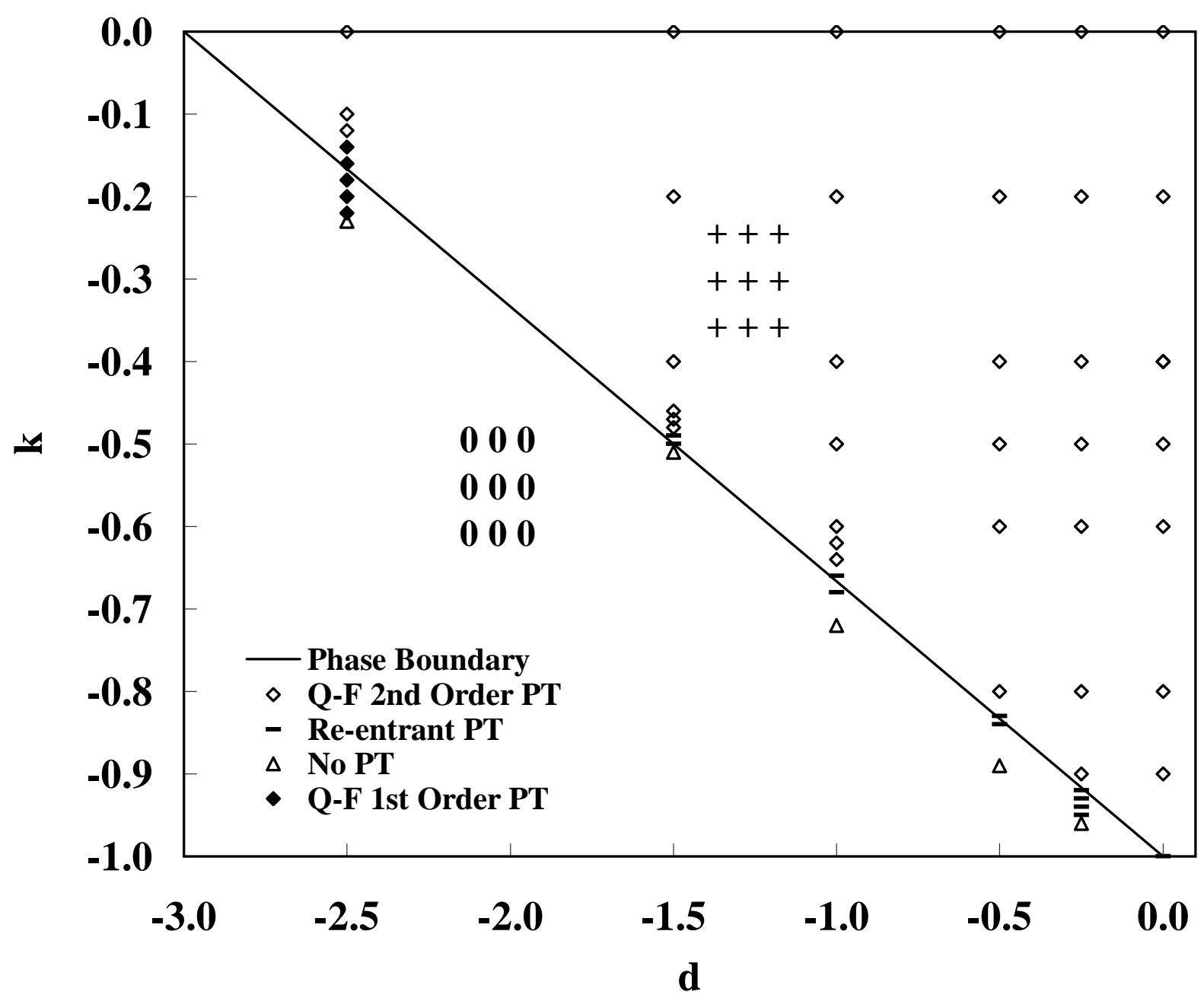



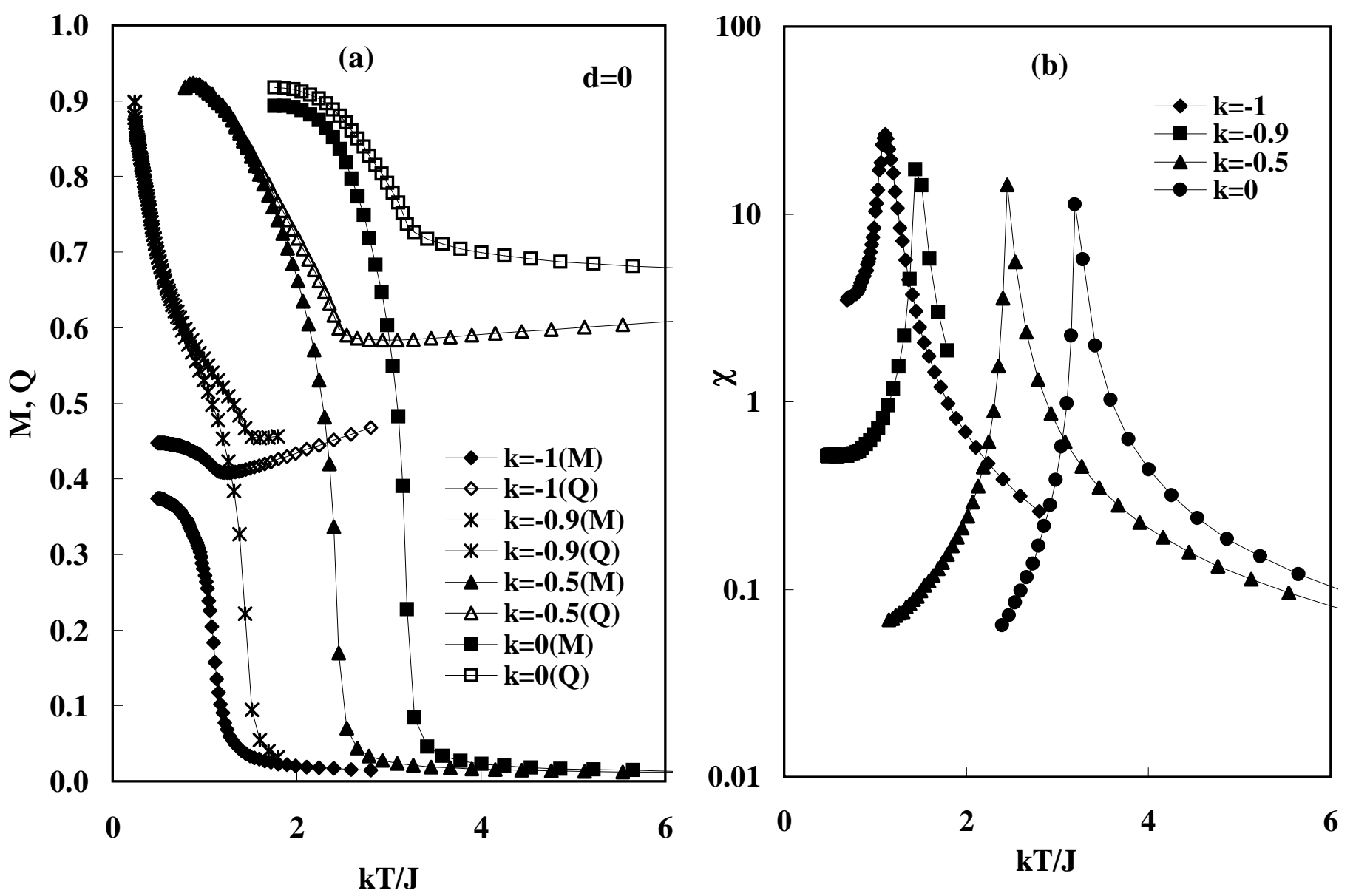

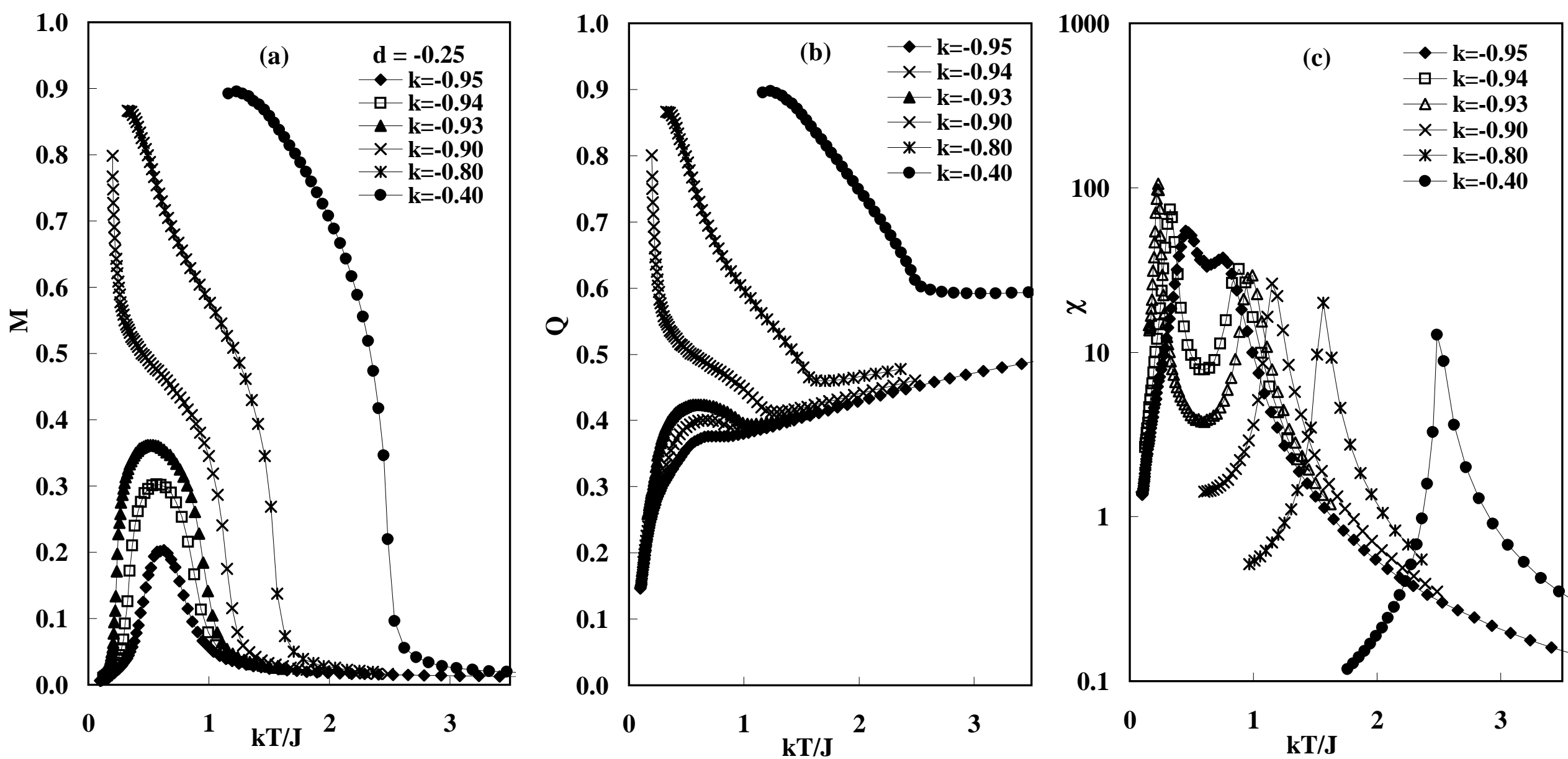

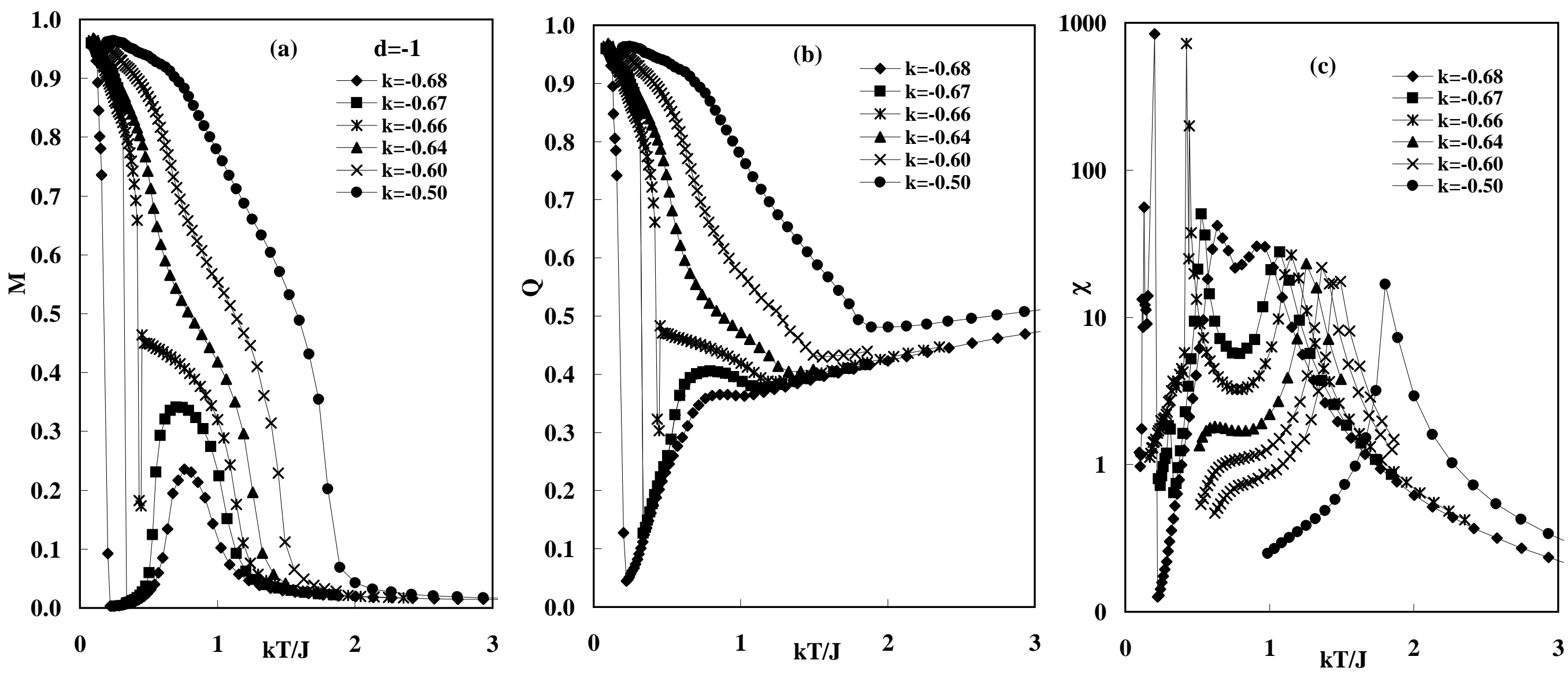

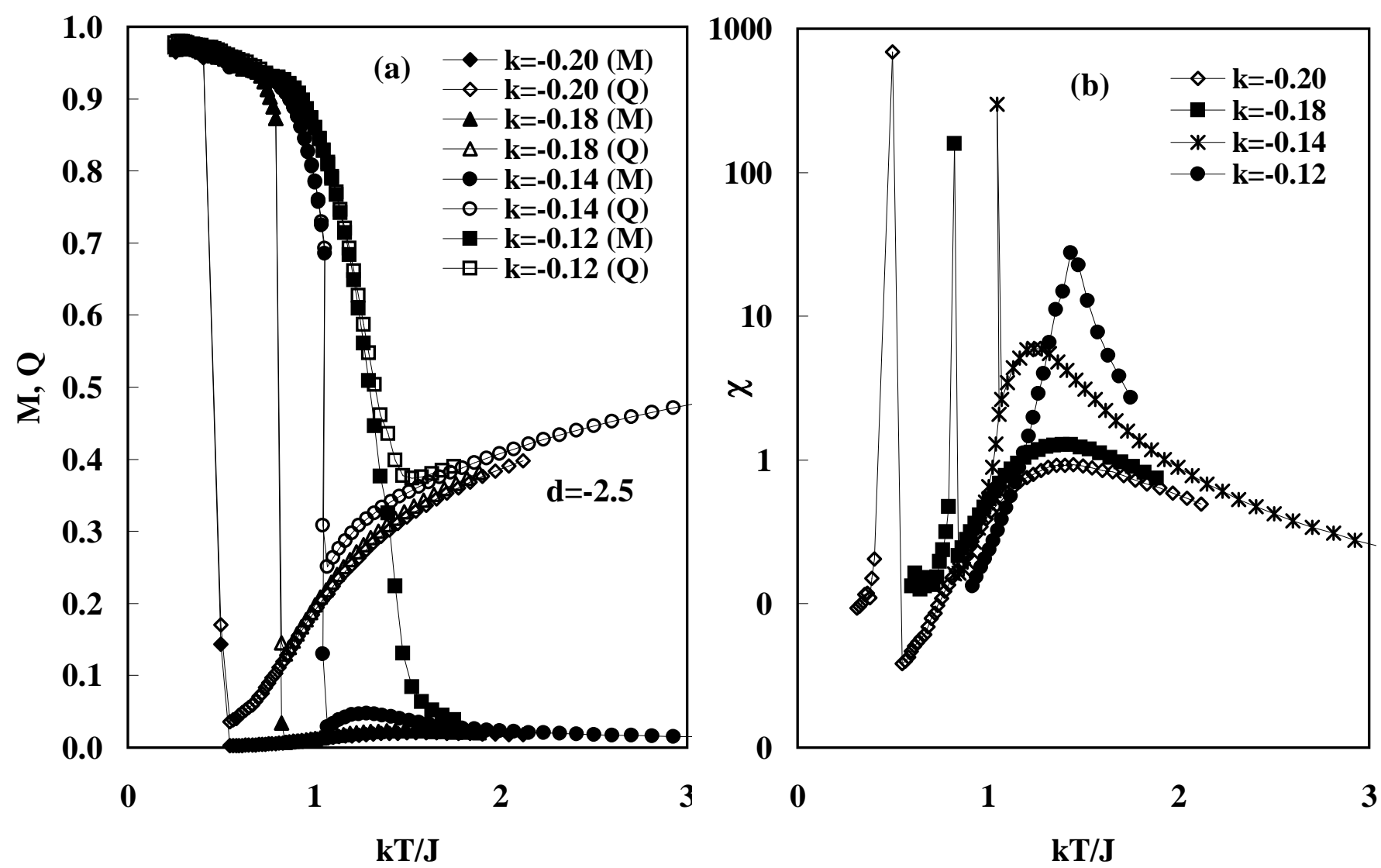


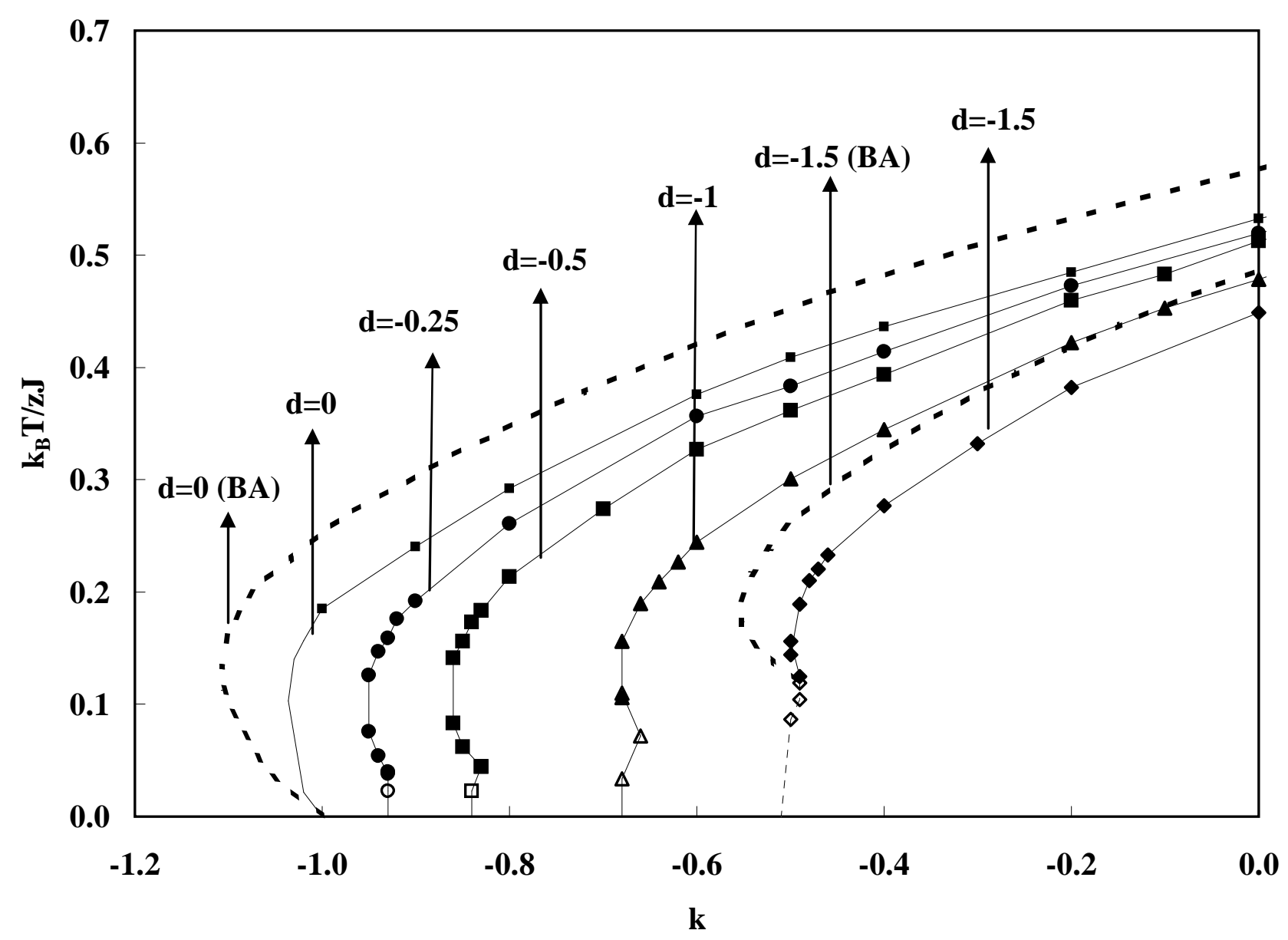




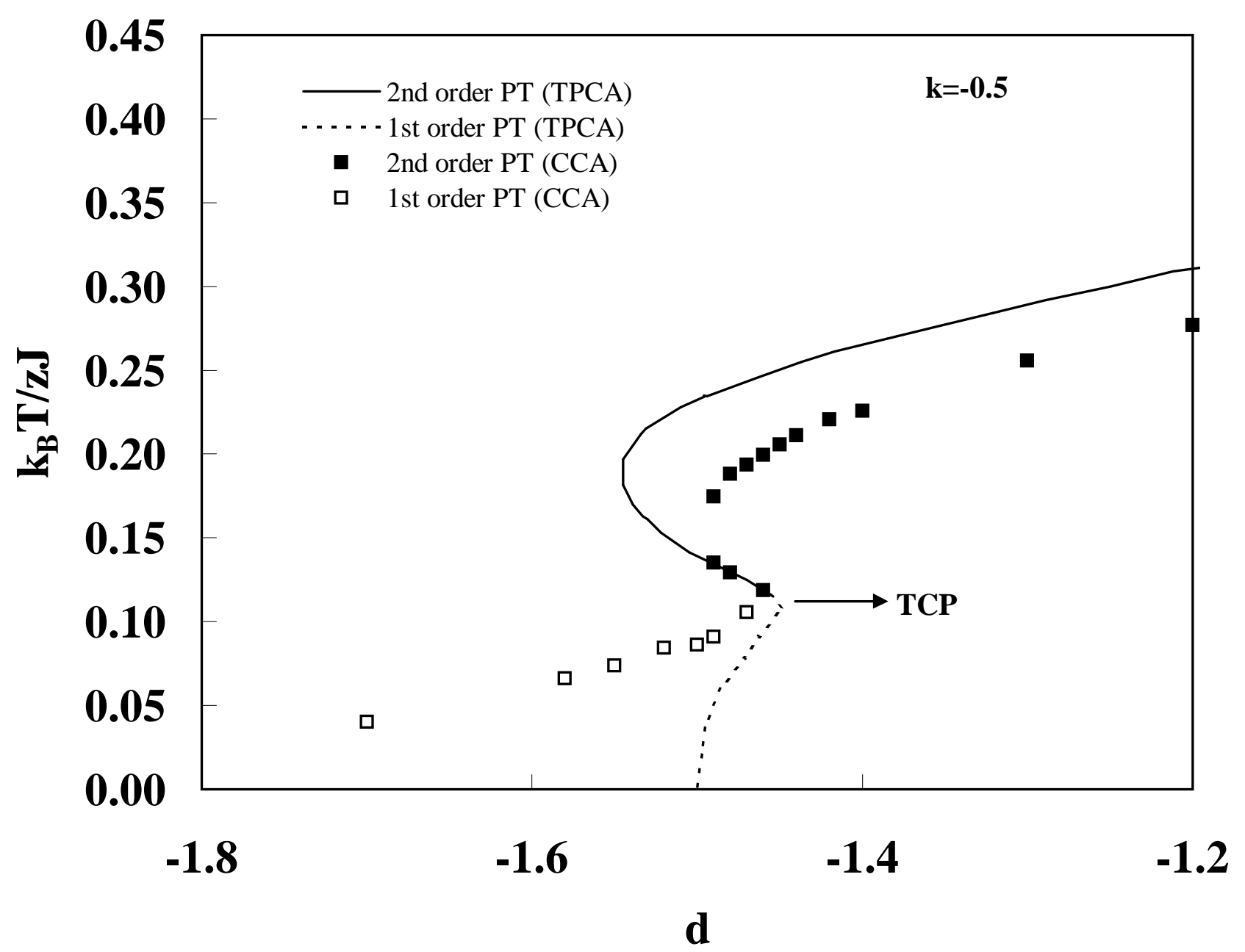

